\title{
AS ESPÉCIES DE COPEPODA (CRUSTACEA: ERGASILIDAE) PARASITAS DOS FILAMENTOS BRANQUIAIS E NARINAS DE Serrasalmus altispinis (MERCKX, JÉGU E SANTOS, 2000) (CHARACIFORMES - SERRASALMIDAE) EM LAGOS DE VÁRZEA DO BRASIL
}

\author{
Germán A.M. MOREY ${ }^{1}$, José C.O. MALTA ${ }^{1}$ \\ 1 Instituto Nacional de Pesquisas da Amazônia, Laboratório de Parasitologia de Peixes Av. André Araújo 2936, Petrópolis, \\ CEP-69.067-375, Manaus, Amazonas, Brasil. E-mail: germantiss@hotmail.com
}

\begin{abstract}
RESUMO
Quatro espécies de Copepoda foram coletadas em Serrasalmus altispinis provenientes de seis lagos de várzea no estado do Amazonas. Amplexibranchius bryconis e Ergasiilus jaraquensis nas brânquias, Gamidactylus jaraquensis e Rhinergasilus piranhus nas narinas. S. altispinis é um novo hospedeiro para estes parasitas. A. bryconis e E. jaraquensis são registrados pela primeira vez parasitando a um peixe da família Serrasalmidae. A. bryconis foi a espécie dominante nas brânquias e a que apresentou os maiores índices parasitários. Nas narinas G. jaraquensis foi o parasita dominante. Houve correlação negativa significativa (p $<0,05)$ entre o comprimento de $S$. altispinis e a abundância de copépodes nas brânquias. Nas narinas houve correlação positiva não significativa entre o comprimento dos hospedeiros e a abundância de copépodes. Foi ampliada a distribuição destes parasitas em um novo hospedeiro.
\end{abstract}

PALABRAS CLAVE: parasitos de peixes; Amplexibranchius bryconis; Ergasiilus jaraquensis; Gamidactylus jaraquensis; Rhinergasilus piranhus.

\section{THE SPECIES OF COPEPODA (CRUSTACEA: ERGASILIDAE) PARASITES OF GILL FILAMENTS AND NOSTRILS OF Serrasalmus altispinis (MERCKX, JÉGU E SANTOS, 2000) (CHARACIFORMES: SERRASALMIDAE) OF CENTRAL AMAZON FLOODPLAIN LAKES, BRAZIL}

\begin{abstract}
Four species of Copepoda were collected in Serrasalmus altispinis from six floodplain lakes, Amazonas State: Amplexibranchius bryconis and Ergasilus jaraquensis were collected in the gills and Gamidactylus jaraquensis and Rhinergasilus piranhus in the nostrils. S. altispinis is a new host for these parasites. A. bryconis e E. jaraquensis are recorded for the first time parasitazing a fish from the family Serrasalmidae. $A$. bryconis was the dominant species in the gills and with higher rates of parasites. In the nostrils G. jaraquensis was the dominant parasite. There was a significant $(\mathrm{p}>0.05)$ negative correlation between the length of $S$. altispinis and abundance of parasites in the gills. In the nostrils there was a non significant positive correlation between the lengh of the hosts and the abundance of parasites. There was expanded the distribution of these parasites in a new host.
\end{abstract}

KEYWORDS: fish parasites; Amplexibranchius bryconis; Ergasiilus jaraquensis; Gamidactylus jaraquensis; Rhinergasilus piranhus. 


\section{INTRODUÇÃO}

O gênero Serrasalmus é distribuído através dos trópicos da América do Sul, este da cordilheira dos Andes, sendo abundantes nas bacias dos rios: Orinoco, Solimões - Amazonas, Paraná/Paraguai e São Francisco e habitando uma ampla variedade de corpos de água (Goulding, 1980). São caracterizados pela natureza predadora altamente especializada e o diversificado hábito alimentar presente no desenvolvimento ontogenético variando desde zooplâncton, insetos a tecidos carnosos de peixes e outros animais (Goulding, 1980; MachadoAllison \& Fink, 1996).

Serrasalmus altispinis é uma espécie simpátrica com S. rhombeus. O corpo em S. altispinis é levemente mais comprimido e profundo que em $S$. rhombeus. S. altispinis é caracterizado pela presença de quatro supraneurals (cinco em $S$. rhombeus). $S$ altispinis possui a serra mais profunda que $S$. rhombeus. Esta espécie foi descrita na bacia Uatumã (Amazonas, Brasil) (Merckx et al., 2000)

O nome "altispinis" provem do latin altus (altura) e spina (espinho), faz referência à altura da serra observada nesta espécie (Merckx et al., 2000).

A classe Copepoda tem aproximadamente 1900 espécies de parasitas de peixes (Eiras, 1994). Eles podem se aderir aos filamentos braquiais, fossas nasais e tegumento. Consequentemente, algumas estruturas foram especializadas para a fixação em diferentes órgãos, e as peças bucais foram adaptados para furar e sugar. Só os indivíduos adultos estão adaptados para o parasitismo (Thatcher, 2006).

Sendo o hospedeiro $S$. altispinis uma espécie relativamente recém-descrita, não existe nenhum trabalho sobre algum Copepoda que a parasite. Por tal motivo o presente trabalho objetivou identificar as espécies de Copepoda que parasitam as brânquias e narinas de $S$. altispinis.

\section{MATERIALE MÉTODOS}

Os exemplares de $S$. altispinis foram capturados em seis lagos de várzea, cinco do rio Solimões: Baixio (03¹7'27,2"S/ 6004'29,6"O) no município de Iranduba; Preto $\left(03^{\circ} 21^{\prime} 17,1^{\prime \prime S} / 60^{\circ} 37^{\prime} 28,6^{\prime \prime} \mathrm{O}\right) \mathrm{em}$ Iranduba; Ananá $\left(03^{\circ} 53^{\prime} 54,8^{\prime \prime} \mathrm{S} / 61^{\circ} 40^{\prime} 18,4^{\prime \prime} \mathrm{O}\right)$ em Anori; Araçá (S034ㄴ $04,3^{\prime \prime} \mathrm{S} / 62^{\circ} 21^{\prime}$ 25,9" O) em Codajás; Maracá (0350'32,8"S/ 62³4'32,4"O) em Coari e um lago do rio Purus, São Tomé $\left(03^{\circ} 49^{\prime}\right.$ $39,0^{\prime \prime} \mathrm{S} / 61^{\circ} 25^{\prime} 24,6^{\prime \prime} \mathrm{O}$ ), durante o período de fevereiro de 2013 a dezembro de 2013,

Foram realizadas oito expedições, quatro por ano durante as estações de cheia, vazante, seca e enchente. Os peixes foram capturados com redes de espera de $20 \mathrm{~m}$ de comprimento por $2 \mathrm{~m}$ de altura com malhas variando de $30,40,50,60,70,80,90$ e $100 \mathrm{~mm}$ entre nós adjacentes. As redes ficaram na água 10 horas em cada lago no período diurno, com duas despescas.

No campo os peixes foram identificados, pesados e medidos. As brânquias e narinas foram removidas e preservadas em frascos de vidro preenchidos com água aquecida a $70^{\circ} \mathrm{C}$ e formol $4 \%$. Os frascos com as amostras de cada peixe foram rotulados com as informações correspondentes.

As brânquias e narinas foram analisadas no Laboratório de Parasitologia de Peixes do Instituto de Pesquisas da Amazônia (LPP - INPA) em Manaus. Sob microscópio estereoscópio os copépodos foram retirados dos filamentos branquiais e narinas, e conservados em álcool $70 \%$. Para o estudo morfológico das espécies de Copepoda, lâminas permanentes foram preparadas segundo o método de Eosina-Orange-G.

A identificação das espécies de Copepoda foi baseada nas características morfológicas e foram utilizados os trabalhos de Thatcher \& Robertson (1982); Thatcher \& Paredes (1985); Thatcher \& Boeger (1984) e Boeger \& Thatcher (1988).

Os descritores ecológicos: intensidade média; abundância média e prevalência foram calculados de acordo com Bush et al. (1997). O status comunitário das infracomunidades parasitárias foi classificado segundo Bush \& Holmes (1986): espécies centrais (presentes em mais de dois terços dos hospedeiros), espécies secundárias (em um a dois terços do hospedeiro) e espécies satélites (em menos de um terço do hospedeiro).

Foi calculado o Coeficiente de Dominância (CD). Este coeficiente mede a porcentagem de uma espécie em relação ao conjunto da infracomunidade parasitária para todos os hospedeiros examinados (Serra-Freire, 2002).

Para analisar as correlações existentes entre o comprimento dos hospedeiros e a abundância de copépodes em brânquias e narinas foi utilizado teste de Spearman $(r s)$. Todos os valores foram considerados significativos quando $\mathrm{p}<0,05$.

\section{RESULTADOS}

Sessenta exemplares de $S$. altispinis de seis lagos de várzea da Amazônia central foram capturados e examinados. Foram coletados nas brânquias e narinas 196 espécimens de Copepoda. Nas brânquias 136 de Amplexibranchius bryconis Thatcher \& Paredes, 1985; e 20 de Ergasilus jaraquensis Thatcher \& Robertson, 1982; nas 
narinas 22 Gamidactylus jaraquensis Thatcher \& Boeger (1984); e um Rhinergasilus piranhus Boeger \& Thatcher(1988).

Amplexibranchius bryconis foi à espécie que apresentou os maiores índices parasitários e o maior número de indivíduos (Tabela 1 ).

A espécie dominante nas brânquias foi $A$. bryconis apresentando o 94,2\% do total das espécies registradas para este órgão. Nas narinas $G$. jaraquensis foi dominante com $95,6 \%$ do total das espécies. O status comunitário das infracomunidades de Copepoda de S. altispinis teve a A. bryconis como a única espécie com status secundário (prevalência $>33 \%$ ), sendo $E$. jaraquensis, $G$. jaraquensis e $R$. piranhus espécies com status satélite (Tabela 1).

Houve uma correlação negativa significativa entre o comprimento dos hospedeiros e a abundância de copépodes parasitas das brânquias $(r s=-0,3788 ; p=0,0066)$ (Figura 1, Tabela 2) e uma correlação positiva não significativa entre o comprimento dos hospedeiros e a abundância de copépodes parasitas das narinas $(r s=0,0705, p=$ 0,6264) (Tabela 2).

Tabela 1. Índices parasitários das espécies de Copepoda parasitas de Serrasalmus altispinis capturados em lagos de várzea, do rio Solimões na Amazônia Central. $\mathrm{PE}=$ peixes examinados; $\mathrm{PP}=$ peixes parasitados; $\mathrm{P}(\%)=$ Prevalência; I = Intensidade parasitaria; IM = Intensidade media; AM = abundância média; $C D$ = Coeficiente de dominância.

\begin{tabular}{|c|c|c|c|c|c|c|c|c|}
\hline Espécies & PE & PP & $\mathbf{P} \%$ & I & IM & AM & $\mathrm{CD} \%$ & $\begin{array}{c}\text { Status } \\
\text { comunitário }\end{array}$ \\
\hline \multicolumn{9}{|l|}{ Brânquias } \\
\hline A. bryconis & 60 & 21 & 35 & $163(1-63)$ & $7,8 \pm 12$ & 2,8 & 94,2 & Secundária \\
\hline E. jaraquensis & 60 & 10 & 17 & $10(1-2)$ & $1,0 \pm 0,33$ & 0,2 & 5,8 & Satélite \\
\hline \multicolumn{9}{|l|}{ Narinas } \\
\hline G. jaraquensis & 60 & 10 & 17 & $22(1-5)$ & $2,2 \pm 1,5$ & 0,4 & 95,6 & Satélite \\
\hline R. piranhus & 60 & 1 & 1,67 & 1 & 1 & 0,02 & 4,4 & Satélite \\
\hline
\end{tabular}

Tabela 2. Valores do coeficiente de correlação de Spearman $(r s)$ entre o comprimento de Serrasalmus altispinis e a abundância de Copepoda parasitas das brânquias e narinas.

\begin{tabular}{|c|c|c|}
\hline & rs & $\boldsymbol{p}$ \\
\hline \multicolumn{3}{|c|}{ Espécies de Copepoda das brânquias } \\
\hline Comprimento VS abundância & $-0,3788$ & 0,0066 \\
\hline \multicolumn{3}{|c|}{ Espécies de Copepoda das narinas } \\
\hline Comprimento VS abundância & 0,0705 & 0,6264 \\
\hline
\end{tabular}




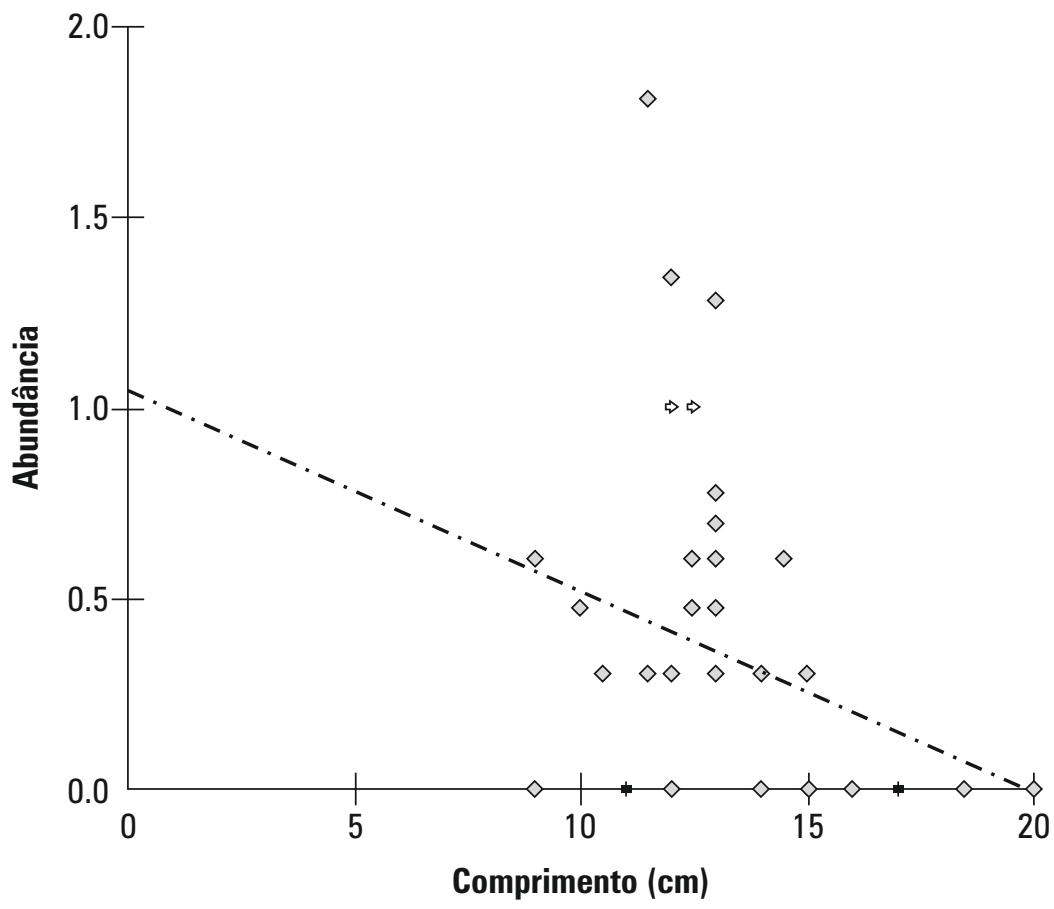

Figura 1. Coeficiente de correlação de Spearman $(r s)$ entre o comprimento de Serrasalmus altispinis e a abundância de copépodes das brânquias. $(r s=-0,3788 ; p=0,0066)$.

\section{DISCUSSÃO}

Amplexibranchius bryconis foi descrito dos filamentos branquiais de Brycon cephalus (sinonímia de B. amazonicus Spix \& Agassiz, 1829) na cidade de Iquitos no Peru (Thatcher \& Paredes 1985b). Morey et al. (2015) registraram pela primeira vez no Brasil o copépode A. bryconis parasitando as brânquias de Potamorhina latior (Spix \& Agassiz, 1829). Neste trabalho é feito o terceiro registro de ocorrência de A. bryconis, o segundo para o Brasil e um novo hospedeiro é conhecido: S. altispinis.

Ergasilus jaraquensis Thatcher \&Robertson, 1982 foi descrito dos filamentos branquiais de Semaprochilodus insignis (Jardine, 1841) do rio Solimões próximo à cidade de Manaus (Thatcher \& Robertson, 1982). Este parasita foi registrado também em Prochilodus lineatus (Valenciennes, 1836) e em P. latior (Morey et al., 2015). Neste trabalho $S$. altispinis é registrada como um novo hospedeiro para E. jaraquensis.

Morey et al. (2015) trabalhando em lagos de várzea da Amazônia Central no Brasil registraram nas brânquias de $P$. latior os copépodes $A$. bryconis $\mathrm{e}$ E. jaraquensis. No presente estudo trabalhando nos mesmos lagos de várzea foram também identificados $A$. bryconis e E. jaraquensis, reforçando assim a presença e a coabitação destes parasitas nestes ambientes.

Rhinergasilus piranhus foi citado parasitando as fossas nasais de P. nattereri (Boeger \& Thatcher, 1988; Varella, 1992; Morais, 2012) indicando a especificidade do parasita tanto pelo hospedeiro quanto para o local de fixação. Embora $R$. piranhus foi considerado um parasita raro com baixos índices parasitários já que só foi registrado um indivíduo em um peixe, este estudo constituí o primeiro registro deste copepodo em $S$. altispinis ampliando assim o número de hospedeiros.

Rhinergasilus piranhus é observado usualmente nos seus hospedeiros junto com outros gêneros como Gamidactylus e Gamispatulus (Varella \& Malta, 1995). Os resultados do presente estudo confirmam o enunciado destes autores já que este parasita foi encontrado juntamente com indivíduos de Gamidactylus jaraquensis. 
Gamidactylus jaraquensis foi descrito pela primeira vez parasitando as fossas nasais de Semaprochilodus insignis no rio Amazonas, próximo a Manaus (Varella, 1992). Fischer et al. (2003), registraram o parasita G. jaraquensis parasitando as narinas de C. macropomum na região de Coari/Tefé. Assim mesmo Lacerda et al. (2007) estudando as fossas nasais de peixes da região do Paraná-Brasil, registraram baixos índices parasitários para os copépodos G. jaraquensis e $R$. piranhus. Estes resultados indicam que estes parasitas mantém um número baixo de indivíduos em média nos seus hospedeiros, limitados possivelmente pelo pequeno espaço disponível dentro da narina.

Atualmente existem poucos registros de copépodes parasitas em hospedeiros da família Serrasalmidae, tendo como registro para peixes de água doce do Brasil os parasitas Brasergasilus bifurcatus Santos, Thatcher \& Brasil-Sato, 2007 nos hospedeiros Pygocentrus piraya e Serrasalmus brandtii; Brasergasilus sp. em P. piraya; Ergasilus yumaricus Malta \& Varella, 1995 em Pygocentrus nattereri, Serrasalmus eigenmanni e Serrasalmus rhombeus; Gamidactylus jaraquensis Thatcher \& Boeger, 1984d em Colossoma macropomum; Gamispatulus schizodontis Thatcher \& Boeger, 1984d em Serrasalmus maculatus e Serrasalmus marginatus; Miracetyma piraya Malta, 1993e em $P$. nattereri; Rhinergasilus piranhus Boeger \& Thatcher, $1988 \mathrm{em}$ P. nattereri; Rhinergasilus sp em $P$. piraya. A. bryconis e E. jaraquensis são registrados pela primeira vez parasitando a um peixe da família Serrasalmidae, ampliando assim o número de copépodes parasitas para peixes desta família.

Os padrões de correlação entre o comprimento do peixe e a riqueza, a intensidade e a abundância de parasitos têm correlações positivas fracas não significativas ou mesmo correlações negativas. Isto ocorre devido à regulação dependente de intensidade (limitação de tamanho e conteúdo de nutrientes). Essa regulação é mais intensa em peixes de maior tamanho do que nos menores (Poulin \& Morand, 1999).

As correlações são influenciadas por restrições biológicas (fisiologia e imunologia) e ecológicas (dinâmica de distribuição) das populações hospedeiras, eventos estocásticos e/ou interações bióticas como competição, regulação dependente de intensidade, inimigos naturais e dieta. Tais restrições evitam altas intensidades, abundância e riqueza de espécies parasitas nos peixes (Alves \& Luque, 2006).
Morey et al. (2015) encontraram uma correlação negativa não significativa entre o comprimento de $P$. latior e a abundância de copépodes parasitas das brânquias.

Neste trabalho houve correlação negativa significativa entre o comprimento dos hospedeiros e a abundância parasitaria de copépodes parasitas das brânquias. No entanto para copépodes parasitas das narinas, houve correlação positiva não significativa entre o comprimento dos hospedeiros e a abundância parasitaria. Esses tipos de correlações podem ser atribuídos às estratégias do ciclo de vida de cada espécie, ao grau de especialização dos órgãos de fixação do parasita e a disponibilidade de formas livres infetantes a determinadas faixas etárias da população hospedeira.

\section{REFERÊNCIAS BIBLIOGRÁFICAS}

Alves, D.R.; Luque, J.L. 2006. Ecologia das comunidades de metazoários parasitos de cinco espécies de escombrídeos (Perciformes: Scombridae) do litoral do estado do Rio de Janeiro, Brasil.Revista Brasileira de Parasitologia Veterinária, 15(4): 167-181.

Boeger, W.A.; Thatcher, V.E. 1988. Rhinergasilus piranhus gen. et spp. nov. (Copepoda, Poecilostomatoidea, Ergasilidae) from the nasal cavities of piranha caju, Serrasalmus nattereri (Kner), in the Central Amazon. Proceedings of the Helminthological Society of Washington, 55, 87-90.

Bush, A.O.; Holmes, J.C. 1986b. Intestinal helmints of lesser scaup ducks: na interactive community. Canadian Journal Zoology, 64: 142-152.

Bush, A.O.; Lafferty, K.D.; Lotz, J.M.; Shostak, A.W. 1997. Parasitology meets ecology on its own terms. Journal of Parasitology, 83: 575583.

Fischer, C.; Malta, J.C.O.; Varella, A.M.B. 2003. A fauna de parasitas do Tambaqui, Colossoma macropomum (Cuvier, 1818) (Characiformes: Characidae) do médio rio Solimoes, Estado do Amazonas (AM) e do baixo rio Amazonas, Estado do Pará (PA), e seu potencial como indicadores biológicos. Acta amazônica 33(4): 651-662.

Lacerda, A.C.F.; Takemoto, R.M.; Lizama, M.A.P.; Pavanelli, G.C. 2007. Parasitic copepods in the nasal fossae of five fish species (Characiformes) from the upper Paraná River floodplain, Paraná, Brazil. Acta Scientiarum Biological Sciences, 29(4): 429-435. 
Malta, J.C.O. 1993e. Miracetyma piraya sp. nov. (Copepoda, Poecilostomatoida, Ergasilidae) dos peixes de água doce da Amazônia Brasileira. ActaAmazonica, 23, 261-269.

Malta J.C.O.; Varella A.M.B. 1995. Ergasilus yumaricus sp. n. (Copepoda: Ergasilidae) das brânquias de Pygocentrus natteri (Kner, 1860), Serrasalmus rhombeus (Linnaeus, 1819) e Pristobrycon eingenmanni (Norman, 1929) (Characiformes: Serrasalmidae) da Amazônia brasileira. Acta Amazonica, 25, 93-100.

Merckx, A.; Jégu, M.; Santos, G.M. 2000. Une nouvelle espèce de Serrasalmus (Teleostei: Characidae: Serrasalminae), S. altispinis n. sp., décrite du Rio Uatumã (Amazonas, Brésil) avec une description complémentaire de S. rhombeus (Linnaeus, 1766) du plateau guyanais. Cybium, 24(2): 181-201.

Morais, A.M. 2012. Biodiversidade da piranha vermelha Pygocentrus nattereri (Kner,1858) (Characiformes; Serrasalmidae) e sua avaliação como bioindicadores na Amazônia Central. Teste de doutorado, Manaus, Instituto Nacional de Pesquisas da Amazônia. 243 pp.

Morey, G. A. M.; Moreira, A.C.; Malta, J.C.O. 2015. As espécies de Copepoda (Crustacea: Ergasilidae) parasitas dos filamentos branquiais de Potamorhina latior (Spix \& Agassiz, 1829) (Characiformes: Curimatidae) de lagos de várzea da Amazônica Central. Folia Amazónica. 24 (2): 203-208.

Poulin, R.; Morand, S. 1999. Geographical distances and the similarity among parasite communities of conspecific host populations. Parasitology, 119: 369-374.

Serra-Freire, N.M. 2002. Planejamento e análise de pesquisas parasitológicas. Editora da Universidade Federal Fluminense, Niteroi, Brasil. 199 pp.
Thatcher, V.E. Robertson, B.A. 1982. The parasitic crustaceans of fishes from the Brazilian Amazon, 3. Ergasilus jaraquensis n. sp. (Copepoda: Cyclopidea) from the gills of Semaprochilodus insignis (Schomburgk). Revista Brasileira de Biologia, 42, 503-513.

Thatcher, V.E.; Boeger, W.A. 1984d. The parasitic crustaceans of fishes from the Brazilian Amazon, 13. Gamidactylus jaraquensis gen. et sp. nov. (Copepoda: Poecilostomatoida: Vaigamidae) from the nasal fossae of Semaprochilodus insignis (Schomburgk). Amazoniana, 8, 421-426.

Thatcher, V.E.; Paredes, V. 1985b. A parasitic copepod, Amplexibranchius bryconis gen. et sp. nov. (Ergasillidae: Acusicolinae), from an Amazonian fish and remarks on the importance of leg morphology in this subfamily. Amazoniana, 9, 205-214.

Thatcher, V.E. 2006. Amazon fish parasites. $2^{\mathrm{a}} \mathrm{ed}$ Pensoft Publishe, Sofia, Moscow. 508 pp.

Varella, A.M.B. 1992. Copépodos (Crustacea) parasitas das fossas nasais de peixes, coletados na região de Rondônia, Brasil. Tese de Doutorado, Instituto de Biociências do Campus de Rio Claro, Universidade Estadual Paulista —Júlio de Mesquita Filhol, Rio Claro, São Paulo. 105pp.

Varella, A.M.B; Malta, J.C.O. 1995. Gamydactylus hoplius sp. n. (Copepoda, Poecilomastidea, Vaigamidae) das fossas nassais de Hoplias malabaricus (Bloch, 1794) (Characiformes. Erythrynidae) da Amazônia brasileira. Acta Amazônica, 25 (3): 281-288.

Recibido: 18 de Febrero del 2016

Aceptado para publicación: 22 de Abril del 2016 